\title{
Early tumor cavitation with regorafenib in metastatic colorectal cancer: A case report
}

\author{
KENTA KAWASAKI $^{1}$, YASUO HAMAMOTO ${ }^{2}$, MASAYUKI ADACHI ${ }^{1}$, \\ TAKANORI KANAI $^{1}$ and HIROMASA TAKAISHI ${ }^{2}$ \\ ${ }^{1}$ Division of Gastroenterology and Hepatology, Department of Internal Medicine; ${ }^{2}$ Keio Cancer Center, \\ Keio University School of Medicine, Shinjuku-Ku, Tokyo 160-8587, Japan
}

Received November 24, 2014; Accepted October 12, 2015

DOI: $10.3892 / \mathrm{ol} .2015 .3905$

\begin{abstract}
Tumoral cavity formation is a characteristic phenomenon reported in anti-angiogenic therapy in lung lesions. A 57-year-old male with multiple pulmonary metastases from colorectal cancer treated with an oral tyrosine kinase inhibitor, regorafenib, exhibited a characteristic cavity formation after the first two cycles. The decrease in the size of tumors was calculated as $38 \%$, and there were associated decreases in the serum concentrations of the tumor markers carcinoembryonic antigen and CA19-9. After eight cycles of treatment, the cavity gradually disappeared through filling-in. This unique morphological response is not only reported in lung cancer but also in liver metastasis in colorectal cancer. However, the association between morphological changes including cavity formation and clinical benefit remains controversial. Pulmonary hemorrhage and pneumothorax are well-known consequences of cavitation, as reported with the other anti-angiogenic inhibitors. Early tumor cavitation in lung metastasis may demonstrate the predictive potential of regorafenib in colorectal cancer, although it is necessary to be mindful of toxicity.
\end{abstract}

\section{Introduction}

The formation of cavities in tumors characteristically occurs following anti-angiogenic therapy for malignant lung lesions; however, cavitation is rarely observed in colorectal cancer. Angiogenesis has been a therapeutic target according to its importance in cancer development, and various anti-angiogenic agents are in use in current cancer treatment. Thus far, several studies have reported a frequency of $\sim 20 \%$ cavity formation following anti-angiogenic therapy (1-3).

Correspondence to: Dr Hiromasa Takaishi, Keio Cancer Center, Keio University School of Medicine, 35 Shinanomachi, Shinjuku-Ku, Tokyo 160-8587, Japan

E-mail: takaishi@z6.keio.jp

Key words: regorafenib, colorectal cancer, lung metastasis, cavity formation
Regorafenib is an oral multikinase inhibitor that targets a broad range of angiogenic, stromal and oncogenic kinases, and is approved for the treatment of colorectal cancer (4). In standard colorectal cancer therapy, regorafenib is used as salvage therapy and, according to the CORRECT trial, median overall survival is 6.4 months (4). Side effects observed in clinical trials of regorafenib were manageable, and the major toxicities were hand-foot skin reactions, diarrhea, hypertension and fatigue (4). Cavity formation following reforafenib treatment was reported for the first time as a correspondence following a phase III trial (5), but the typical manifestation was not demonstrated. The generally accepted mechanism for the development of cavitation is rapid tumor growth, which outstrips the tumor blood supply $(1,2)$. At present, the clinical benefit of cavitation remains unclear $(2,3)$. Here, we report the case of a 57-year-old male who was treated with regorafenib as a single agent for pulmonary metastases from colorectal cancer and developed extensive characteristic cavitation followed by filling-in. Written informed consent was obtained from the patient.

\section{Case report}

A 57-year-old male was diagnosed with sigmoid colorectal cancer [stage IIA (T3N0M0), KRAS mutation-positive, G12D] and underwent anterior resection. The patient's medical history included subarachnoid hemorrhage at the age of 39 , which was treated by surgery, and hypertension at the same age. His medication was Candesartan $8 \mathrm{mg} /$ day.

One year after surgery, multiple lung metastases were diagnosed and the patient commenced treatment with S-1, oxaliplatin and bevacizumab. He received this regimen for 21 cycles and was then switched to capecitabine, irinotecan and bevacizumab due to progressive disease (PD). After receiving 14 cycles of this combination, the patient was referred to the Keio Cancer Center, Tokyo, Japan, for salvage line treatment. A computed tomography (CT) scan of the chest revealed several large masses in the lungs (longest diameter, $36 \mathrm{~mm}$; Fig. 1A-C) and the patient commenced regorafenib $160 \mathrm{mg} /$ day.

Following the first two cycles, a notable response was demonstrated on CT, with shrinkage and characteristic cavitation being visible in all metastatic deposits (Fig. 2A-C). The 


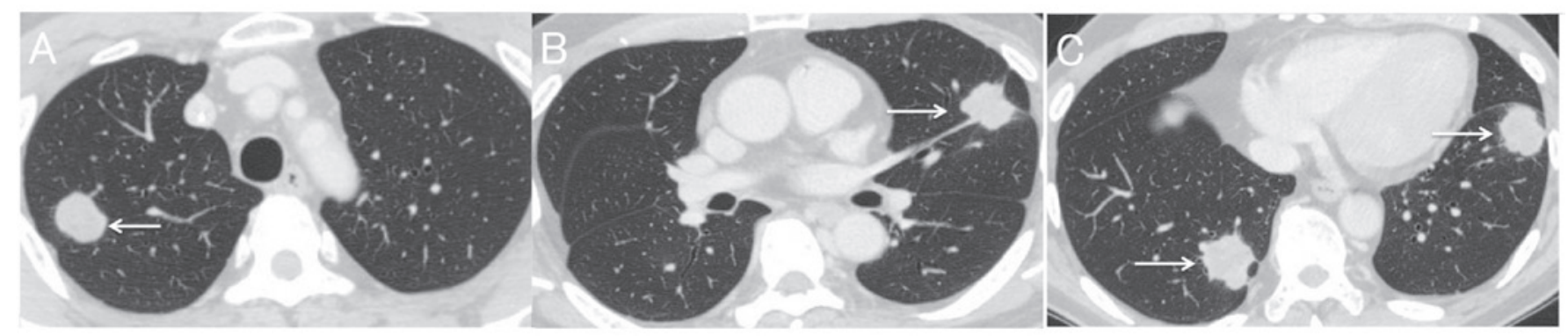

Figure 1. (A-C) Chest computed tomography images prior to treatment with regorafenib demonstrating presence of multiple metastases in the lungs (arrows). Longest diameter, $36 \mathrm{~mm}$ (June 2013).
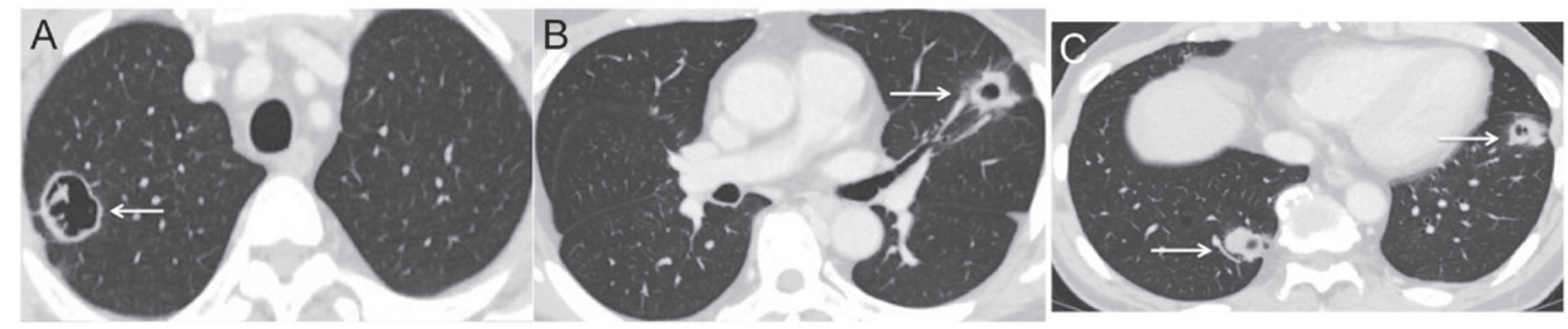

Figure 2. (A-C) The multiple metastatic tumor lesions (arrows) decreased in size and demonstrated characteristic tumor cavitation following two cycles of regorafenib (160 mg/day) (August 2013).
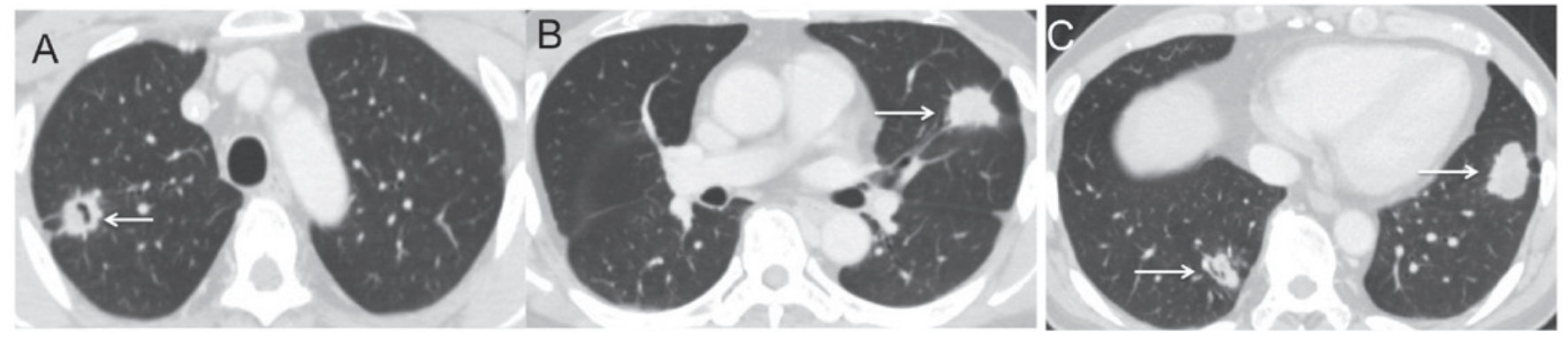

Figure 3. (A-C) The multiple metastatic tumor lesions (arrows) continued to shrink but now represent progressive disease as the cavitation is no longer present (January 2014).

decrease in the size of tumors was calculated as $38 \%$, and there were associated decreases in the serum concentrations of the tumor markers carcinoembryonic antigen and CA19-9. The patient was evaluated as having a partial response. The toxicities experienced were hand-foot syndrome and hypertension, which were tolerated, and chest pain and hemoptysis were not observed. After the patient had received eight cycles, the metastatic lesions had enlarged by $27 \%$ and the cavities had disappeared through filling-in, resulting in an evaluation of PD (Fig. 3A-C).

\section{Discussion}

In the present study, we report the case of a patient with a characteristic manifestation of multiple pulmonary metastases of colorectal cancer that responded to regorafenib with cavity formation.

The generally accepted mechanism for the development of cavitation is a tumor growth so rapid that it outstrips the tumor blood supply, forming central necrosis and inhibiting tumor-associated vasculature $(1,2)$. The cavity formation caused by regorafenib may be attributable to the same mechanisms. With regard to the correlation between clinical benefit and cavitation, there are no significant differences in progression-free or overall survival between subjects with and without cavitation reported in lung cancer $(2,3)$.

This unique morphological response not only occurs in lung cancer but also in liver metastasis in colorectal cancer. However, liver metastasis is replaced by fibrosis and necrosis, and is observed in $<5 \%$ of all tumors (6). Significantly, morphological changes have been reported in association with pathological response and overall survival in liver metastasis of colorectal cancer (6). However, the association between these morphological changes and clinical benefit remains controversial.

The well-known consequences of cavitation include pulmonary hemorrhage and pneumothorax treated with anti-angiogenic therapy $(7,8)$; however, the association between hemoptysis and cavitation is controversial $(2,3,6)$. 
Given that regorafenib caused cavitation in our case, bleeding events and pneumothorax could have occurred.

In conclusion, early tumor cavitation in lung metastasis demonstrates the predictive potential of regorafenib in colorectal cancer. Attention should also be paid to the possibility of hemoptysis, pneumothorax and chest pain occurring with cavitation, which are associated with the use of other anti-angiogenic inhibitors $(7,8)$. Research into the correlation between cavitation and efficacy is ongoing as a subgroup analysis in the CORRECT trial (4). Further investigation of the toxicity profile is required in the post-marketing setting.

\section{References}

1. Crabb SJ, Patsios D, Sauerbrei E, Ellis PM, Arnold A, Goss G, Leighl NB, Shepherd FA, Powers J, Seymour L and Laurie SA: Tumor cavitation: impact on objective response evaluation in trials of angiogenesis inhibitors in non-small-cell lung cancer. J Clin Oncol 27: 404-410, 2009.

2. Nishino M, Cryer SK, Okajima Y, Sholl LM, Hatabu H, Rabin MS, Jackman DM and Johnson BE: Tumoral cavitation in patients with non-small-cell lung cancer treated with antiangiogenic therapy using bevacizumab. Cancer Imaging 12: 225-235, 2012.
3. Marom EM, Martinez CH, Truong MT, Lei X, Sabloff BS, Munden RF, Gladish GW, Herbst RS, Morice RC, Stewart DJ, et al: Tumor cavitation during therapy with antiangiogenesis agents in patients with lung cancer. J Thorac Oncol 3: 351-357, 2008.

4. Grothey A, Van Cutsem E, Sobrero A, Siena S, Falcone A, Ychou M, Humblet Y, Bouché O, Mineur L, Barone C, et al; CORRECT Study Group: Regorafenib monotherapy for previously treated metastatic colorectal cancer (CORRECT): An international, multicentre, randomised, placebo-controlled, phase 3 trial. Lancet 381: 303-312, 2013.

5. Ricotta R, Sartore-Bianchi A, Verrioli A, Vanzulli A and Siena S: Regorafenib for metastatic colorectal cancer. Lancet 381: 1537, 2013

6. Chun YS, Vauthery JN, Boonsirikamchai P, Maru DM, Kopetz S, Palavecino M, Curley SA, Abdalla EK, Kaur H, Charnsangavej C and Loyer EM: Association of computed tomography morphologic criteria with pathologic response and survival in patients treated with bevacizumab for colorectal liver metastases. JAMA 302: 2338-2344, 2009.

7. Cho YJ, Murgu SD and Colt HG: Bronchoscopy for bevacizumab-related hemoptysis. Lung Cancer 56: 465-468, 2007.

8. Verschoor AJ and Gelderblom H: Pneumothorax as adverse event in patients with lung metastases of soft tissue sarcoma treated with pazopanib: a single reference centre case series. Clin Sarcoma Res 4: 14, 2014. 